\title{
Delegate Action
}

National Cancer Institute

\section{Source}

National Cancer Institute. Delegate Action. NCI Thesaurus. Code C73926.

To entrust to another. 